\title{
Compression defects in different reconstructions from phase-shifting digital holographic data
}

\author{
Emmanouil Darakis, ${ }^{1, *}$ Thomas J. Naughton, ${ }^{2,3}$ and John J. Soraghan ${ }^{1}$ \\ ${ }^{1}$ Institute for Communications and Signal Processing, Department of Electronic and Electrical Engineering, \\ University of Strathclyde, G1 1XW, Glasgow, UK \\ ${ }^{2}$ Department of Computer Science, National University of Ireland, Maynooth, County Kildare, Ireland \\ ${ }^{3}$ RFMedia Laboratory, University of Oulu, Oulu Southern Institute, Vierimaantie 5, 84100 Ylivieska, Finland \\ ${ }^{*}$ Corresponding author: emmdarakis@ieee.org
}

Received 14 December 2006; revised 7 March 2007; accepted 12 March 2007; posted 14 March 2007 (Doc. ID 78106); published 6 July 2007

\begin{abstract}
Three principal strategies for the compression of phase-shifting digital holograms (interferogram domain-, hologram domain-, and reconstruction domain-based strategies) are reviewed and their effects in the reconstruction domain are investigated. Images of the reconstructions are provided to visually compare the performances of the methods. In addition to single reconstructions the compression effects on different depth reconstructions and reconstructions corresponding to different viewing angles are investigated so that a range of the 3D aspects of the holograms may be considered. Although comparable at low compression rates, it is found that depth and perspective information is degraded in different ways with the different techniques at high compression rates. A hologram of an object with sufficient details at different depths is used so that both parallax and depth effects can be illustrated. (C) 2007 Optical Society of America

OCIS codes: $\quad 050.5080,090.1760,090.2880,100.2000,110.3000$.
\end{abstract}

\section{Introduction}

Phase-shifting interferometry (PSI) [1] digital holography is a $3 \mathrm{D}$ imaging technique where the complex light distribution at the plane of the recording camera can be calculated from several interference patterns corresponding to different reference light phases. Compared with other digital holography techniques this method has the advantage of increased reconstruction quality as the unwanted zero-order and twin image terms are eliminated [2]. This enables the use of an in-line recording setup reducing the recording sensor resolution requirements.

As several interferograms have to be recorded, the use of the method is practically limited to static scenes as the light distribution from the object should remain constant over the recording. Another disadvantage of the method is that the resulting wavefront consists of complex floating point numbers occupying a large amount of storage space. Recently introduced

0003-6935/07/214579-08\$15.00/0

(C) 2007 Optical Society of America color PSI holography [3] requires that one wavefront for each of the primary colors (blue, green, and red) is used thus increasing the complexity. Practical applications of PSI holography, on the other hand, require that data volumes are minimized either for more efficient storage or for faster transmission. As a result, PSI holographic data compression is of the significant importance.

The speckle nature of the PSI holograms of macroscopic reflective objects places constraints on the possible compression efficiency $[4,5]$. Several techniques for PSI holographic data compression have been studied. Initially uniform scalar quantization of the PSI hologram values followed by lossless coding of the quantized data was suggested [5-7]. The method has also been applied following wavelet decomposition of the wavefront [8] and extended to use k-means instead of uniform quantization showing increased compression efficiency [9]. Each of these methods operates on the complex wavefront at the plane of the camera. Compression on the interference patterns has also been suggested [10-13] offering promising results while using common image compression tech- 
niques. Finally, compression of PSI holographic data at the reconstruction plane [13,14] using Fresnelets $[13,15]$ has been investigated as offering increased compression performance.

In this paper we study the visual effects of the coding methods on the reconstructed images. Apart from a single reconstruction at a given distance we investigate the compression effects on reconstructions from the same hologram at different distances as well as on reconstructions corresponding to different observation angles. To this end we apply the methods under investigation on a hologram with adequate details on different depths so that both the depth and parallax effects can be clearly seen. The compression effects are investigated at both low and higher compression rates.

The paper is organized as follows. In Section 2 the PSI recording procedure is introduced, followed by a discussion in Section 3 on the compression approaches that have been considered. Section 4 introduces the experimental setup that has been used to capture the holographic data, discusses the experiment that has been done, and finally illustrates and analyzes the results that have been obtained. Section 5 concludes the paper.

\section{Phase-Shift Interferometry Digital Holography}

A simplified recording setup for capturing PSI holograms is shown in Fig. 1. The object to be recorded is positioned at a distance $d_{0}$ from the camera. A laser source of wavelength $\lambda$ is used to illuminate the object. The light beam is split by a beam splitter into two parts. One of them is used to illuminate the objects to be recorded. The light reflected from the object reaches the CCD sensor through the second beam splitter. Its distribution at the plane of the camera can be expressed as

$$
U(x, y)=A_{0}(x, y) \exp \left[i \varphi_{0}(x, y)\right],
$$

where $A_{0}(x, y)$ and $\varphi_{0}(x, y)$ are the amplitude and the phase of the wave respectively. The second part passes through a half-wave and a quarter-wave retardation plates. By appropriately adjusting the fast and slow axes of these plates steps of $\phi=0, \pi / 2, \pi$, and $3 \pi / 2$ can be added to the phase of the reference beam. Through the second beam splitter the phasemodulated reference beam reaches the camera where it interferes with the object beam to form the inter-

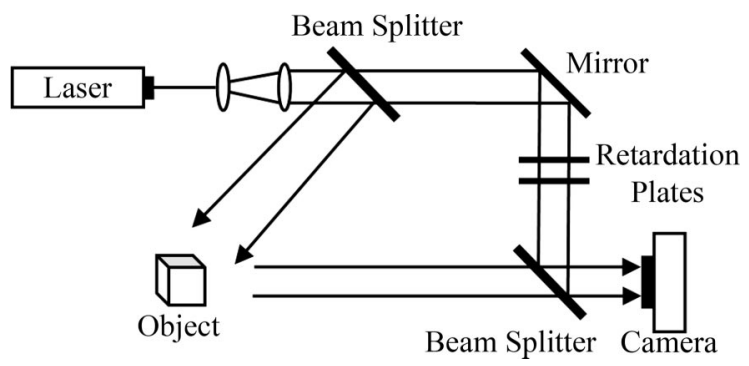

Fig. 1. Simplified PSI hologram recording setup. ferogram $I(x, y ; \phi)$. Four interferograms corresponding to the four phase steps are recorded. These interferograms can be expressed as

$$
I(x, y ; \phi)=\left|U_{0}(x, y)+U_{R}(x, y ; \phi)\right|^{2},
$$

where $U_{R}(x, y ; \phi)$ is the phase-modulated reference wave. Using the PSI algorithm [1] the wavefront $U(x, y)$ at the plane of the camera can be calculated from the recorded interference patterns as

$$
\begin{aligned}
U(x, y)= & \frac{1}{4 A_{R}}\{[I(x, y ; 0)-I(x, y ; \pi)] \\
& +i[I(x, y ; \pi / 2)-I(x, y ; 3 \pi / 2)]\},
\end{aligned}
$$

where $A_{R}$ is the amplitude of the reference wave. In the case of a plane normally incident reference wave it can be assumed that $A_{R}=1$. The complex wavefront calculated by Eq. (3) is also called a PSI hologram or Fresnel field. Notice that each of the interference patterns is an in-line hologram, and as a result to avoid confusion we refer to the complexvalued (wavefronts) holograms as PSI holograms and to the real-valued holograms (interference patterns) as interference patterns.

The PSI hologram has been calculated at the plane of the camera. To reconstruct the wavefront at the plane of the object the PSI hologram has to be propagated to a distance $d_{0}$. This can be done using the Fresnel-Kirchhoff integral [16] defined as

$$
\begin{aligned}
U_{d}\left(x^{\prime}, y^{\prime}\right)= & \frac{1}{\tau^{2}} \int_{-\infty}^{+\infty} \int_{-\infty}^{+\infty} U(x, y) \exp \left\{\frac { i \pi } { \tau ^ { 2 } } \left[\left(x-x^{\prime}\right)^{2}\right.\right. \\
& \left.\left.+\left(y-y^{\prime}\right)^{2}\right]\right\} \mathrm{d} x \mathrm{~d} y \\
= & \left(U * G_{\tau}\right)\left(x^{\prime}, y^{\prime}\right),
\end{aligned}
$$

where

$$
G_{\tau}(x, y)=\frac{1}{\tau^{2}} \exp \left[\frac{i \pi}{\tau^{2}}\left(x^{2}+y^{2}\right)\right],
$$

$\tau=\sqrt{\lambda d}, d$ is the propagation distance, $*$ denotes convolution, and the constant phase exponential factors have been neglected for simplicity.

In Fig. 2 the images of the corresponding data on the three stages of the procedure are shown. Figure 2(a) shows an interference pattern. Fig. 2(b) shows the magnitude of the PSI hologram at the camera plane, and Figs. 2(c) and 2(d) show the magnitude of the propagated wavefront at distances 263 and $277 \mathrm{~mm}$, respectively.

\section{Compression Approaches}

A. Interference Patterns Compression

Successful compression of PSI holographic data by coding the interference patterns has been reported [10-13]. With this method each of the recorded in- 


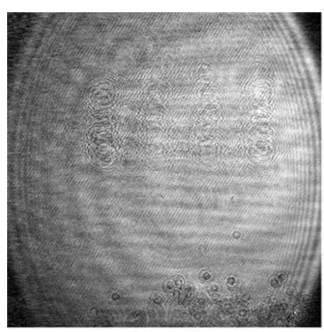

(a)

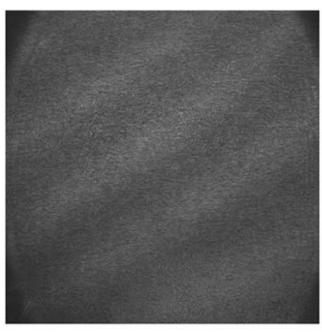

(b)

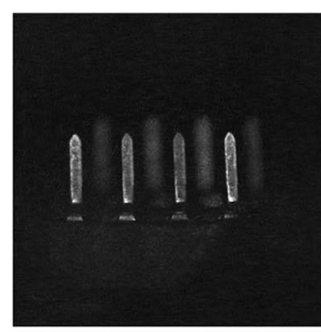

(c)

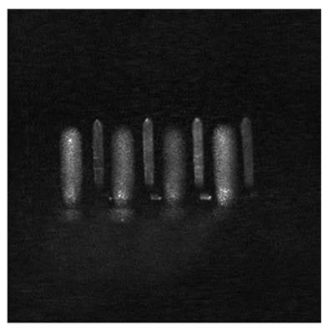

(d)

Fig. 2. PSI data at different stages: (a) Captured interference pattern, (b) magnitude of the PSI hologram, (c) reconstruction at 263 mm, and (d) at $277 \mathrm{~mm}$.

terference patterns $I(x, y ; \phi)$ is individually compressed before the PSI algorithm described by Eq. (3). These interferograms are real-valued grayscale images so the usual image coding techniques can be used for their compression. Standard baseline JPEG [17] and JPEG2000 [18] as well as set partitioning in hierarchical trees (SPIHT) [19] coding have been used. These compression methods were designed for natural, real-world images and not for interference patterns. Nevertheless, it has been shown [11-13], and it is experimentally demonstrated in this paper, that the fringe information of the interference patterns can be effectively retained by these forms of compression and hence usual image compression techniques can still be used for the compression of holographic data.

To obtain the complex wavefront at the camera plane the coded interferograms have to be decoded and combined using Eq. (3). The propagation of the resulting wavefront to the desired distance using Eq. (4) yields reconstructions from the compressed data. Herein we investigate only baseline JPEG2000 compression of the interferograms as the most representative method.

\section{B. Camera Plane Compression}

The initial compression approaches of PSI holographic data were based on camera plane compression [5-7]. With these approaches the PSI hologram $U(x, y)$, which is calculated using Eq. (3), is coded. Several different methods have been applied including uniform scalar quantization followed by lossless coding of the quantized data [5-7], wavelet decomposition of $U(x, y)$ and quantization of the wavelet coefficients [8], k-means quantization and lossless coding [9], companding [20], and others.

In this paper we will investigate only uniform scalar quantization at the camera plane and not other more advanced techniques such as k-mean quantization [9], which offer higher compression efficiency for an increased computational cost [13] or companding [20], which reduces the computational cost. The effect of these methods on the reconstructed images is expected to be equivalent to the effect of the uniform scalar quantization as they also operate on the quantization principle.

In the case of uniform scalar quantization at the camera plane the bit depth of the real and the imag- inary parts of the complex wavefront values are reduced to $m$-bits per sample using

$$
\hat{U}(x, y)=\operatorname{round}\left[U(x, y) \times\left(2^{m-1}-1\right) / U_{\max }\right],
$$

where $U_{\max }=\max (\max \{|\operatorname{Im}[U(x, y)]|\}, \max \{\mid \operatorname{Re}[U$ $(x, y)] \mid\})$. Following this, the quantized data are coded with a lossless algorithms such as [21] to further increase the compression rate. To obtain a reconstruction from the compressed data, lossless decoding is performed, and the decoded data are multiplied by $U_{\max }$ to bring them back to their original range. Propagating the dequantized complex wavefront using Eq. (4) yields the reconstruction from the decoded data.

The main advantage in compressing the PSI hologram at the camera plane using quantization is that the coding is performed pixel-by-pixel and hence the introduced error affects a single pixel at a time contrary to transform-based techniques where usually blocks of samples are affected. In this way the errors introduced to the speckle pattern are minimal. However, the PSI hologram is expected to have high spectral density as according to Eq. (3) it is a linear combination of interferograms, which contain fine fringe details. This can be seen in Fig. 2(b) where the values of the PSI hologram at the plane of the camera appear to be randomly distributed and have high dynamic range. This reduces the effectiveness of the lossless coding and the compression performance of these approaches [13]. Another disadvantage of the method is that it suffers from compression inflexibility in the sense that the compression rate and the introduced error cannot be controlled as the effectiveness of the lossless coder cannot be predetermined.

\section{Compression at the Reconstruction Plane}

The propagated wavefronts in Figs. 2(c) and 2(d) appear more structured than the PSI hologram itself. This indicates that compression at the reconstruction domain may be advantageous [13-15]. The apparent structure at the reconstruction plane is expected to increase the effectiveness of the lossless coder and hence an overall higher compression performance is expected. Two methods, which are performed on the reconstruction plane, have been presented. The first uses the method that has been applied at the camera 
plane directly at the reconstruction plane [14] and the second uses Fresnelet decomposition [15].

Fresnelets are waveletlike bases specially designed for digital holography applications [22]. They are a combination of b-spline wavelets and the Fresnel transform. One-dimensional b-splines of degree $n$ are defined as the $(n+1)$-fold convolution of a rectangular pulse [23]

$$
\beta^{n}(x)=\frac{\beta^{0} * \ldots * \beta^{0}(x),}{n+1 \text { times }}
$$

where

$$
\beta^{0}(x)=\begin{array}{ll}
1, & -1 / 2<x<1 / 2 \\
1 / 2, & |x|=1 / 2 \\
0, & \text { otherwise. }
\end{array}
$$

B-splines can be used to generate a multiresolution analysis of $L_{2}(\mathbb{R})$ [24]. More specifically it has been shown that a two-scale relation of the form

$$
\beta^{n}\left(\frac{x}{2}\right)=\sum_{k} h(k) \beta^{n}(x-k),
$$

where $h(k)$ is the binomial filter $h(k)=\frac{1}{2^{n}}\left(\begin{array}{c}n+1 \\ k\end{array}\right)$ holds. Also semiorthogonal b-spline wavelets of the form

$$
\left\{\psi_{j, k}^{n}=2^{(-j / 2)} \psi^{n}\left(2^{-j} x-k\right)\right\}_{j, k \in Z},
$$

where

$$
\psi^{n}\left(\frac{x}{2}\right)=\sum_{k} g(k) \beta^{n}(x-k),
$$

with $g(k)$ being the quadrature mirror filter of $h(k)$ form Riesz bases of $L_{2}(\mathbb{R})$.

F-splines are Fresnel-transformed b-spline bases. By using the properties of the Fresnel transform (linearity, shift invariance, and scaling) [22] the family of functions $\left\{\left(\widetilde{\left.\psi_{j, k}\right)_{\tau}}=G_{\tau} * \psi_{j, k}^{n}\right\}_{j, k \in Z}\right.$ can be derived. F-splines can be obtained by convolving $G_{\tau}(x)$ with the b-spline $\beta^{n}(x)$ as

$$
\tilde{\beta}_{\tau}{ }^{n}(x)=\left(\beta^{n} * G_{\tau}\right)(x) .
$$

The f-splines can then be used as appropriate bases to decompose the wavefront to different scales. More specifically the two-scale relation corresponding to Eq. (9) becomes

$$
\tilde{\beta}_{\tau / 2}{ }^{n}\left(\frac{x}{2}\right)=\sum_{k} h(k) \tilde{\beta}_{\tau}^{n}(x-k) .
$$

Also, the generating functions corresponding to the b-splines wavelets of Eq. (11) have the form

$$
\tilde{\psi}_{\tau / 2}{ }^{n}\left(\frac{x}{2}\right)=\sum_{k} g(k) \tilde{\beta}_{\tau}^{n}(x-k)
$$

It can be noticed that the generating function changes from scale to scale. As a result, Fresnelets are not valid scaling functions since they do not fulfill the two-scale relation condition. However the embeddedness of the decomposition is preserved as it has been shown [22].

B-splines can be extended to $2 \mathrm{D}$ as

$$
\beta^{n}(x, y)=\beta^{n}(x) \cdot \beta^{n}(y),
$$

where $\cdot$ corresponds to the tensor product. Similarly f-splines can be extended to 2D and hence 1D Fresnelet transform can be expanded to 2D. The Fresnelet coefficients of the wavefront $U(x, y)$ at scale $j=1$ can be obtained as

$$
\begin{aligned}
L L\left(\frac{x}{2}, \frac{y}{2}\right) & =U *\left[\tilde{\beta}_{\tau / 2}{ }^{n}\left(\frac{x}{2}, \frac{y}{2}\right) \cdot \tilde{\beta}_{\tau / 2}{ }^{n}\left(\frac{x}{2}, \frac{y}{2}\right)\right] \\
H L\left(\frac{x}{2}, \frac{y}{2}\right) & =U *\left[\tilde{\psi}_{\tau / 2}{ }^{n}\left(\frac{x}{2}, \frac{y}{2}\right) \cdot \tilde{\beta}_{\tau / 2}{ }^{n}\left(\frac{x}{2}, \bar{y}\right)\right] \\
L H\left(\frac{x}{2}, \frac{y}{2}\right) & =U *\left[\tilde{\beta}_{\tau / 2}{ }^{n}\left(\frac{x}{2}, \frac{y}{2}\right) \cdot \tilde{\psi}_{\tau / 2}{ }^{n}\left(\frac{x}{2}, \frac{y}{2}\right)\right] \\
H H\left(\frac{x}{2}, \frac{y}{2}\right) & =U *\left[\tilde{\psi}_{\tau / 2}{ }^{n}\left(\frac{x}{2}, \frac{y}{2}\right) \cdot \tilde{\psi}_{\tau / 2}{ }^{n}\left(\frac{x}{2}, \frac{y}{2}\right)\right] .
\end{aligned}
$$

To decompose the signal to higher scales the $L L$ part of scale $j=1$ has to be further decomposed. Figure 3 shows the magnitude of the Fresnelet coefficients at scale $j=5$. The actual calculations of the convolution can be carried out by evaluating them in the Fourier domain [22]. More details on the implementation of the Fresnelet decomposition can be found in [15].

Owing to the fact that the Fresnel transform is part of the Fresnelet decomposition they correspond to a wavelet decomposition of the reconstruction domain. Following this, any multiresolution-based method can be used for compression. In our experiments we

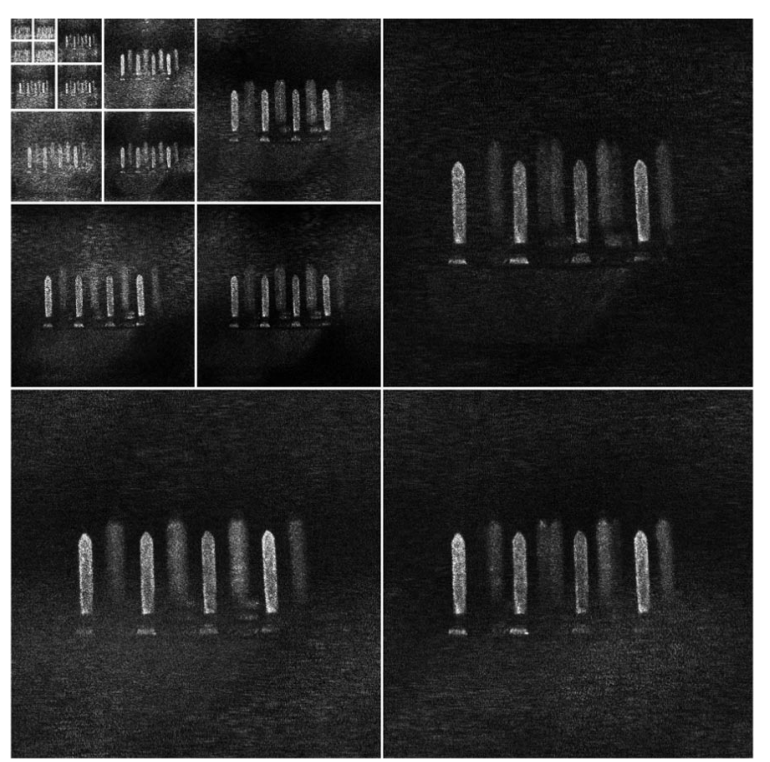

Fig. 3. Magnitude of the Fresnelet coefficients at scale $j=5$. 
have considered SPIHT coding of the Fresnelet coefficients.

The SPIHT algorithm [19] groups Fresnelet coefficients in subband trees and codes them in order of significance. Bits are stored in the bit stream so that an increasingly refined copy of the original image is obtained by time. Information is also stored in the bit stream so that the order of significance can be determined by the decoder. Hence the bit stream produced by the SPIHT algorithm is fully embedded. This means that it can be truncated to any required length, and the remaining part can still yield a reconstruction, which will be optimal in terms of quality for the given bitstream length. In this way the compression rate can be set to any arbitrary value. Other advantages of the SPIHT algorithm include its high encoding and decoding speed and simplicity.

Although reconstruction plane compression is performed on a single reconstruction distance this does not limit the inherent 3D information stored in the PSI holograms because reconstructions at other distances or from different viewing angles can still be obtained [14].

\section{Experimental Setup and Results}

\section{A. Experimental Setup}

To investigate the compression effects of the considered algorithms we applied them on a real PSI hologram we recorded. A similar recording setup to the one shown in Fig. 1 was used. The laser source was radiating a beam at $\lambda=632.8 \mathrm{~nm}$. The recorded scene consisted of a chip positioned upsidedown so that the two rows of the chip pins were positioned approximately perpendicular to the optical axis and at approximate distances $d_{1}=263 \mathrm{~mm}$ and $d_{2}=$ $277 \mathrm{~mm}$. The recording camera had a resolution of $N_{x} \times N_{y}=2048 \times 2032$ pixels with a pixel pitch of $\Delta_{x}=\Delta_{y}=9 \mu \mathrm{m}$. The analysis was carried out using MATLAB. Figure 2 shows the obtained holographic data at different stages of the procedure.

The normalized root mean square (NRMS) metric is used for the quantitative analysis, and it is defined as follows:

$$
\mathrm{NRMS}=\left[\sum_{N_{x}} \sum_{N_{y}}\left[\left|U_{d}\right|^{2}-\left|\hat{U}_{d}\right|^{2}\right]^{2} / \sum_{N_{x}} \sum_{N_{y}}\left[\left|U_{d}\right|^{2}\right]^{2}\right]^{1 / 2},
$$

where $U_{d}$ and $\hat{U}_{d}$ are the propagated wavefronts at the object plane from the original and the compressed data, respectively. The compression rate is calculated as follows:

$$
r=\frac{s}{\hat{s}}
$$

where the $s$ and $\hat{s}$ are the size of the uncompressed and the compressed data files respectively. In our case $s=2 \times N_{x} \times N_{y} \times 8$ bytes $=63.5$ Mbytes as each of the real and imaginary part of the uncompressed complex wavefront consists of $N_{x} \times N_{y}=2048 \times$ 2032 double precision ( 8 bytes) samples.

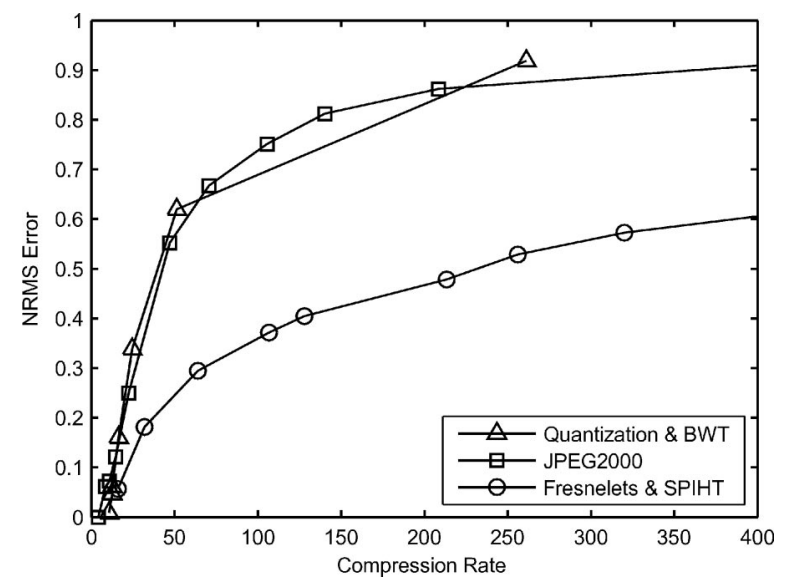

Fig. 4. NRMS numerical results for the investigated coding methods.

\section{B. Single Depth Reconstruction}

For this experiment we implemented three compression schemes, namely:

(i) JPEG2000 compression of the interferograms,

(ii) Uniform scalar quantization of the PSI hologram at the camera plane,

(iii) SPIHT coding of cubic $(n=3)$ Fresnelet coefficients for several compression rates.

In Fig. 4 the numerical results obtained from these methods at different compression rates are shown. The NRMS error and the compression rate have been computed using Eqs. (17) and (18). For experiments at low compression rates we consider approximate rates of 22,16 , and 32 for the JPEG2000, quantization, and SPIHT coding, respectively. For experiments at higher compression rates we consider approximate rates of 209, 261, and 256 for the JPEG2000, quantization, and SPIHT coding of the Fresnelet coefficients, respectively.

\section{Different Depth Reconstruction}

For different depth reconstructions we experiment we have obtained reconstructions from the PSI hologram at the distances 263 and $277 \mathrm{~mm}$. In Fig. 5 the obtained reconstructions are shown for low compression data. Figures 5(a) to 5(d) correspond to reconstructions at $d_{1}$ while Figs. 5(e)-5(h) correspond to reconstructions at $d_{2}$ for the original, JPEG2000, quantization at the camera plane, and SPIHT coding of the Fresnelet coefficients. In Fig. 6 the same reconstructions are shown but from holographic data that have been compressed at the higher rates. In the case of Fresnelet-based coding, the data were coded at distance $d_{1}$, and to obtain the reconstruction at distance $d_{2}$ the procedure described in [14] was used. This involves obtaining the reconstruction at $d_{1}$ and then propagating the wavefront consecutively back to the camera plane $(d=0)$ and then back to $d_{2}$. 


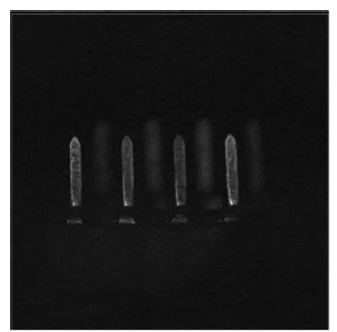

(a)

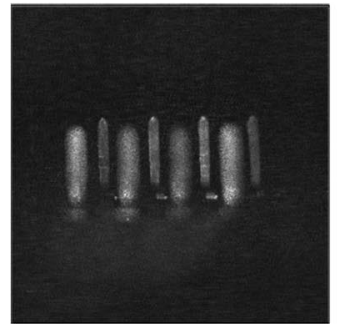

(e)

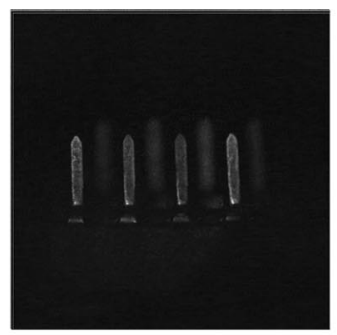

(b)

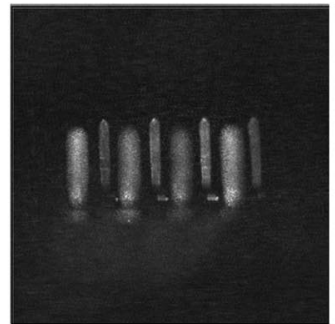

(f)

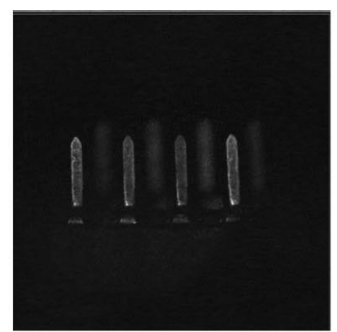

(c)

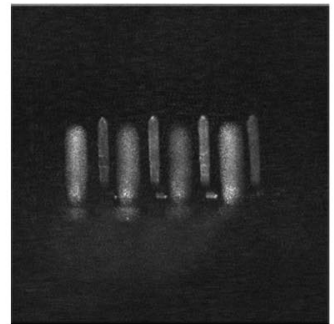

(g)

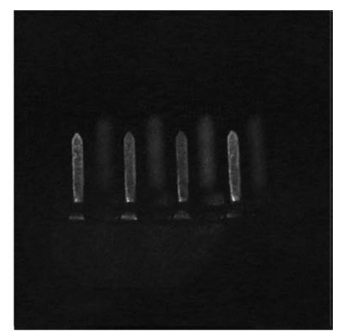

(d)

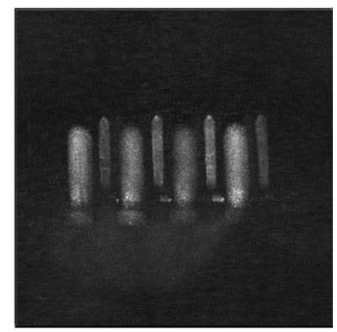

(h)

Fig. 5. Depth effects from low compressed data. (a)-(d) Reconstructions from the original, JPEG2000, quantized, and SPIHT coded data, respectively, for reconstruction distance $263 \mathrm{~mm}$. (e)-(h) Same for distance $277 \mathrm{~mm}$.

\section{Different Viewing Angle Reconstructions}

To investigate the effect of compression on different viewing angles we reconstructed regions from the PSI hologram yielding different viewing angles. More specifically two regions of size $512 \times 2048$ pixels were considered. In one case the left-hand side of the PSI hologram was considered and in the other one the right-hand side region. In Fig. 7 reconstructions corresponding to the left-hand and right-hand sides of the PSI hologram for low compression rates are shown for the different coding methods. Similarly, Fig. 8 shows the same reconstructions from the data coded at a high rate. In the case of Fresnelet-based coding to obtain the different reconstructions the procedure described in [14] was used. This involves reconstructing the whole wavefront at $d_{1}$, and then this wavefront was brought consecutively to the camera plane $(d=0)$, the required portion of it was selected and then it was propagated back to $d_{1}$.

\section{E. Discussion}

From the experimental results shown in Figs. 5 and 7 it is noticed that for low compression rates all the methods yield adequate reconstruction quality in all

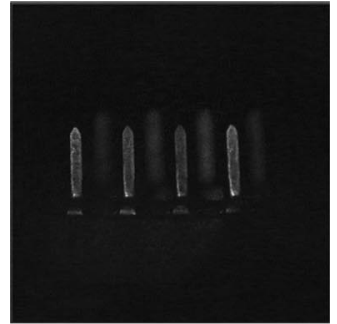

(a)

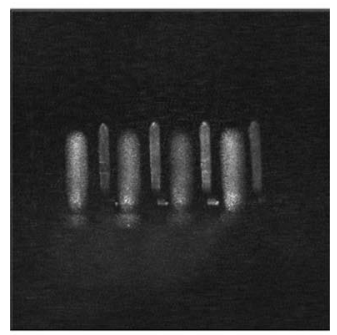

(e)

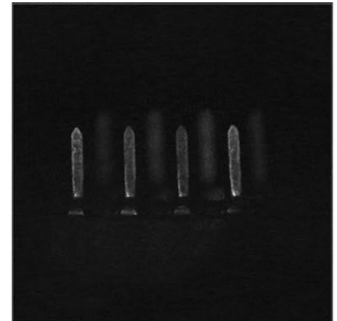

(b)

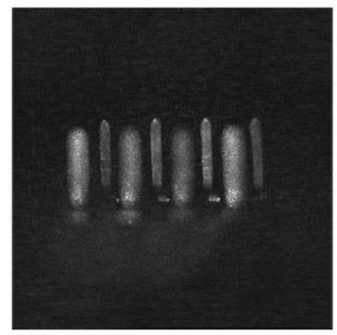

(f)

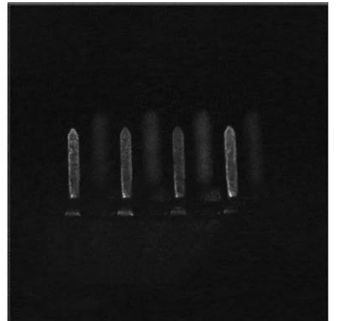

(c)

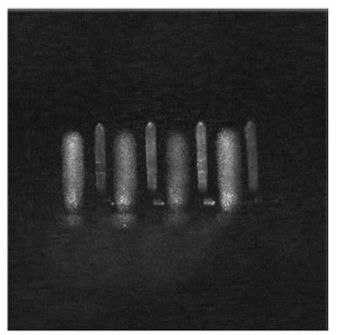

(g)

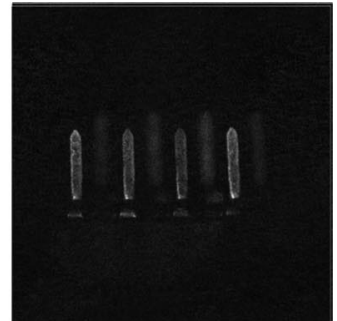

(d)

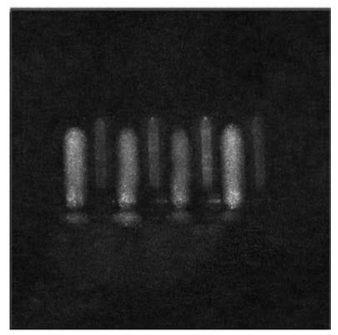

(h)

Fig. 6. Depth effects from highly compressed data. (a)-(d) Reconstructions from the original, JPEG2000, quantized, and SPIHT coded data, respectively, for reconstruction distance $263 \mathrm{~mm}$. (e)-(h) Same for distance $277 \mathrm{~mm}$. 


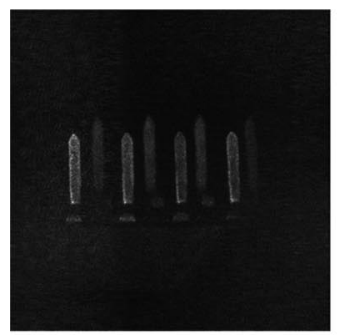

(a)

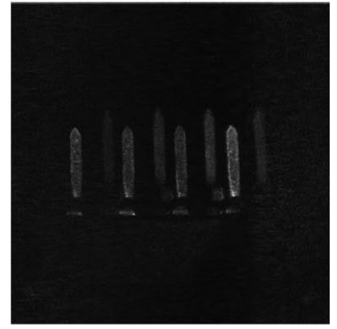

(e)

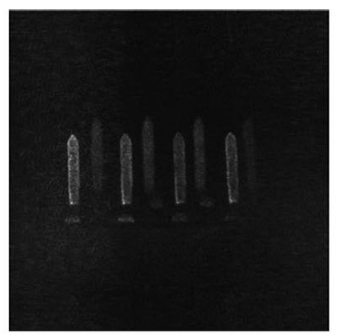

(b)

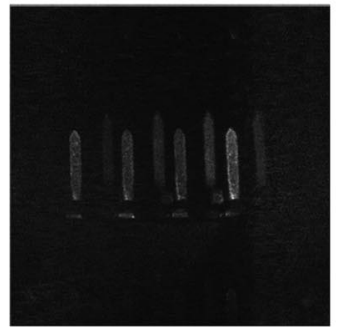

(f)

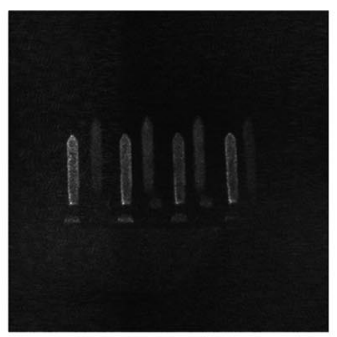

(c)

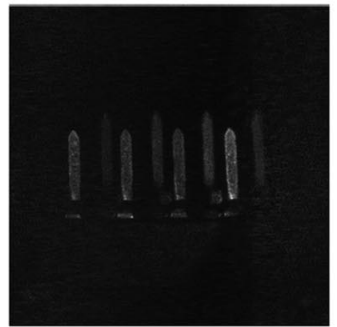

(g)

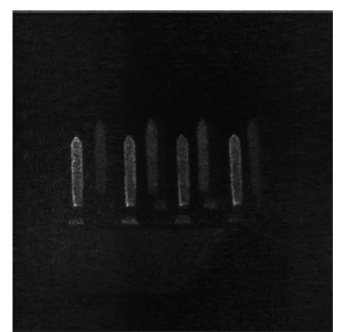

(d)

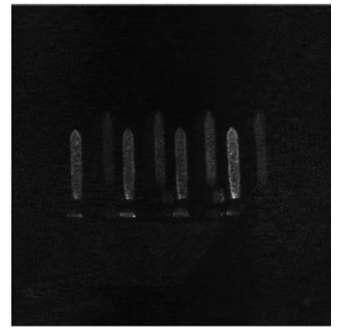

(h)

Fig. 7. Parallax effects from low compressed data. (a)-(d) Corresponding to the left view reconstructions from the original, JPEG2000, quantized, and SPIHT coded data. (e)-(h) Same for a view from the right.

cases. For higher compression rates the reconstruction quality deteriorates, but each of the methods behaves differently as is shown in Figs. 6 and 8 .

More specifically in Figs. 6 and 8 it can be seen that with high rate JPEG2000 coding of the interferograms the reconstruction suffers mainly from an increase in noise as well as ghosting effects, similar to those observed in [25]. These ghosting effects appear only in the case of JPEG2000 compression of the interferograms and not when other image compression techniques such as JPEG or SPIHT are applied.
The main effect of quantization applied on the PSI hologram at the camera plane is an increase of the observed noise compared with the reconstruction from the uncompressed data as it is shown in Figs. 6(c) and 6(g). Also it can be noticed in Fig. 8 that quantization retains more details of the object compared to the other techniques in the case of parallax experiments.

Finally, in the case of the SPIHT coding of the Fresnelet coefficients the main observed effect is that the bright parts of the reconstruction are adequately

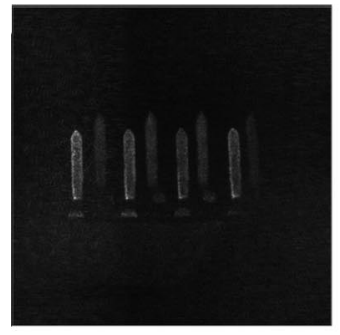

(a)

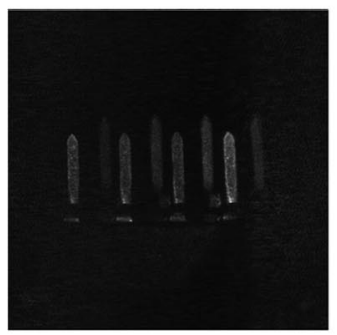

(e)

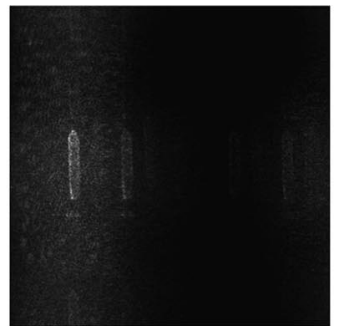

(b)

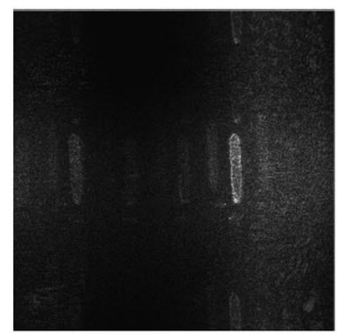

(f)

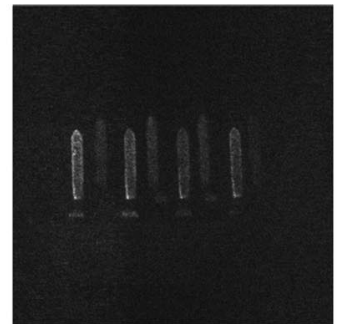

(c)

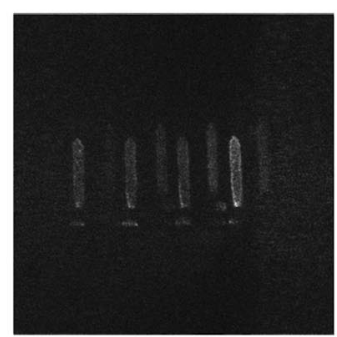

(g)

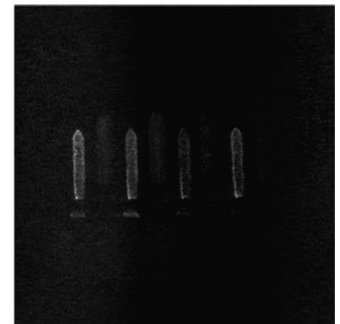

(d)

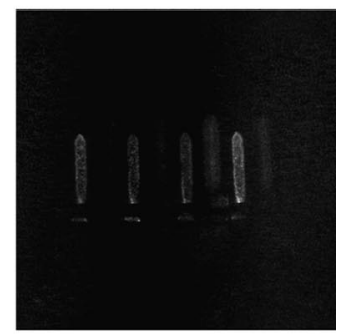

(h)

Fig. 8. Parallax effects from highly compressed data. (a)-(d) Corresponding to the left view reconstructions from the original, JPEG2000, quantized, and SPIHT coded data. (e)-(h) Same for a view from the right. 
retained while the fainter ones are further suppressed as a result of the coding, as can be seen in Figs. 8(d) and 8(h). For high compression rates, and without parallax, the SPIHT coding of the Fresnelet coefficients seems to be more severe than the other techniques when reconstruction depths other than the encoding depth are sought as can be seen in Figs. 6(d) and 6(h). However, interestingly, with this blurring, the noise of the reconstructions is retained low after Fresnelet-based compression indicating that Fresnelets could be used to reduce speckle, as previously proposed [6].

\section{Conclusions}

A study of the visual effects of various PSI holographic data compression techniques on the reconstructions has been presented. The methods have been considered to fall within the following three main compression categories depending on the stage of the PSI reconstruction procedure that they are applied to, namely:

(i) Interference patterns compression,

(ii) Coding of the PSI hologram at the camera plane, and

(iii) Fresnelet-based compression at the reconstruction plane.

From each method we have chosen the most representative technique and applied it to a real PSI hologram with adequate depth details. In addition to the usual single depth reconstruction evaluation of the compression performance we have also considered reconstructions corresponding to different depths as well as from different viewing angles. In this way we observed the effects of the considered methods not only on single reconstructions but on aspects of the whole $3 \mathrm{D}$ recording range of the hologram. The study revealed the different ways in which each of the coding methods affects the reconstructed images at different compression rates.

This work was supported in part by the Greek State Scholarships Foundation, Enterprise Ireland, and Science Foundation Ireland. We thank Yann Frauel, Albertina Castro, and John McDonald for assistance with the optical setup.

\section{References}

1. I. Yamaguchi and T. Zhang, "Phase-shifting digital holography," Opt. Lett. 22, 1268-1270 (1997).

2. U. Schnars and W. P. O. Juptner, "Digital recording and numerical reconstruction of holograms," Meas. Sci. Technol. 13, R85-R101 (2002).

3. I. Yamaguchi, T. Matsumura, and J. Kato, "Phase-shifting color digital holography," Opt. Lett. 27, 1108-1110 (2002).

4. R. Shahnaz, J. F. Walkup, and T. F. Krile, "Image compression in signal-dependent noise," Appl. Opt. 38, 5560-5567 (1999).

5. T. J. Naughton, Y. Frauel, B. Javidi, and E. Tajahuerce, "Compression of digital holograms for three-dimensional object re- construction and recognition," Appl. Opt. 41, 4124-4132 (2002).

6. T. J. Naughton, J. B. McDonald, and B. Javidi, "Efficient compression of Fresnel fields for Internet transmission of threedimensional images," Appl. Opt. 42, 4758-4764 (2003).

7. T. J. Naughton and B. Javidi, "Compression of encrypted three-dimensional objects using digital holography," Opt. Eng. 43, 2233-2238 (2004).

8. A. E. Shortt, T. J. Naughton, and B. Javidi, "Compression of digital holograms of three-dimensional objects using wavelets," Opt. Express 14, 2625-2630 (2006).

9. T. J. Naughton, Department of Computer Science, National University of Ireland, Maynooth, Ireland, A. E. Shortt, and B. Javidi are preparing a manuscript to be called "Nonuniform quantization compression of digital holograms."

10. G. A. Mills and I. Yamaguchi, "Effects of quantization in phase-shifting digital holography," Appl. Opt. 44, 1216-1225 (2005).

11. E. Darakis and J. J. Soraghan, "Compression of interference patterns with application to phase-shifting digital holography," Appl. Opt. 45, 2437-2443 (2006).

12. E. Darakis and J. J. Soraghan, "Compression of phase-shifting digital holography interference patterns," in Photon Management II, J. T. Sheridan and F. Wyrowski, eds., Proc. SPIE 6187, 61870Y (2006).

13. E. Darakis, T. J. Naughton, J. J. Soraghan, and B. Javidi, "Measurement of compression defects in phase-shifting digital holographic data," in Optical Information Systems IV, B. Javidi, D. Psaltis, and H. J. Caulfield, eds., Proc. SPIE 6311, 63110B (2006).

14. E. Darakis and J. J. Soraghan, "Reconstruction domain compression of phase-shifting interferometry digital holograms," Appl. Opt. 46, 351-356 (2007).

15. E. Darakis and J. J. Soraghan, "Use of Fresnelets for phaseshifting digital hologram compression," IEEE Trans. Image Process. 15, 3804-3811 (2006).

16. J. W. Goodman, Introduction to Fourier Optics (Roberts \& Company, 2005).

17. W. B. Pennebaker and J. L. Mitchell, JPEG Still Image Data Compression Standard (Van Nostrand Reinhold, 1993).

18. T. Acharya and P.-S. Tsai, JPEG2000 Standard for Image Compression: Concepts, Algorithms and VLSI Architectures (Wiley, 2005).

19. A. Said and W. A. Pearlman, "A new, fast, and efficient image codec based on set partitioning in hierarchical trees," IEEE Trans. Circuits Syst. Video Technol. 6, 243-250 (1996).

20. A. E. Shortt, T. J. Naughton, and B. Javidi, "A companding approach for nonuniform quantization of digital holograms of three-dimensional objects," Opt. Express 14, 5129-5134 (2006).

21. M. Burrows and D. Wheeler, A Block-Sorting Lossless Data Compression Algorithm (Digital Systems Research Center, Palo Alto, 1994).

22. M. Liebling, T. Blu, and M. Unser, "Fresnelets: new multiresolution wavelet bases for digital holography," IEEE Trans. Image Process. 12, 29-43 (2003).

23. M. Unser, "Splines: a perfect fit for signal and image processing," IEEE Signal Process Mag. 16, 22-38 (1999).

24. M. Unser, P. Thevenaz, and A. Aldroubi, "Shift-orthogonal wavelet bases using splines," IEEE Signal Process Lett. 3, 85-88 (1996).

25. D. Kayser, T. Kreis, and W. Juptner, "Compression of digital holographic data using its electromagnetic field properties," Proc. SPIE 5908, 59080C-59089 (2005). 